\title{
Study of the B[sub s] meson with forthcoming LHC data at CMS
}

\author{
Millán Mejías, B
}

\begin{abstract}
A study of the properties of the Bs meson decays has been done using a full Monte Carlo simulation of the CMS detector. The CMS pixel detector allows a precise measurement of the Bs decay vertices which are displaced from the proton-proton interaction point. This facilitates a time dependent analysis of the angular correlations between the Bs decay products. In particular, the decay Bs- $>(\mathrm{J} / \mathrm{psi}) \mathrm{phi}-$ $>\mathrm{u}+\mathrm{\mu}-\mathrm{K}+\mathrm{K}-$ enables measurements of the width difference between heavy and light mass eigenstates. Furthermore, it permits a measurement of the CP violating phase phis which is sensitive to physics beyond the Standard Model. (C2010 American Institute of Physics
\end{abstract}

DOI: https://doi.org/10.1063/1.3480181

Posted at the Zurich Open Repository and Archive, University of Zurich

ZORA URL: https://doi.org/10.5167/uzh-46082

Conference or Workshop Item

Published Version

Originally published at:

Millán Mejías, B (2009). Study of the B[sub s] meson with forthcoming LHC data at CMS. In: VIII Latin American Symposium on Nuclear Physics and Applications, Santiago, Chile, 15 December 2009 19 December 2009. American Institute of Physics, 264-267.

DOI: https://doi.org/10.1063/1.3480181 


\title{
Study of the $B_{S}$ meson with forthcoming LHC data at CMS
}

\author{
B. Millán Mejías on behalf of the CMS Collaboration \\ Physik-Institut, Universität Zürich, Switzerland
}

\begin{abstract}
A study of the properties of the $B_{s}$ meson decays has been done using a full Monte Carlo simulation of the CMS detector. The CMS pixel detector allows a precise measurement of the $B_{S}$ decay vertices which are displaced from the proton-proton interaction point. This facilitates a time dependent analysis of the angular correlations between the $B_{s}$ decay products. In particular, the decay $B_{s} \rightarrow(J / \psi) \phi \rightarrow \mu^{+} \mu^{-} K^{+} K^{-}$enables measurements of the width difference between heavy and light mass eigenstates. Furthermore, it permits a measurement of the CP violating phase $\phi_{s}$ which is sensitive to physics beyond the Standard Model.
\end{abstract}

Keywords: LHC, CMS, $B_{s}$ meson

PACS: $14.40 . \mathrm{n}$

\section{INTRODUCTION}

The Compact Muon Solenoid (CMS) is one of the multipurpose detectors at the Large Hadron Collider (LHC) at CERN. LHC is a pp collider with the designed center of mass energy of $\sqrt{s}=14 \mathrm{TeV}$. The CMS silicon tracker [1], closest detector to the interaction point, consists of 1440 silicon pixel and 15148 silicon strip detector modules. It is located, together with the electromagnetic and hadron calorimeters, inside a superconducting solenoidal magnet, which provides an axial field of $3.8 \mathrm{~T}$. Outside of the solenoid is the muon system for identifying and triggering on muons and for their trajectory reconstruction in the magnet return yoke.

The pixel detector performance allows precise measurements of the $B_{s}$ meson decay vertices, permitting to measure properties of two $B_{s}$ weak eigenstates $B_{s}^{L}$ and $B_{s}^{H}$ such as differences between their widths $\left(\Delta \Gamma_{s}\right)$ and masses $\left(\Delta m_{s}\right)$. Moreover, the decay $B_{s} \rightarrow$ $(J / \psi) \phi \rightarrow \mu^{+} \mu^{-} K^{+} K^{-}$provides one of the best ways to determine the weak angle $\phi_{s}$, where $\phi_{s}$ is the $B_{s}^{0}$ mixing phase angle (or twice the angle $\beta_{s}$ ) in the unitary triangle.

In the first section mixing in the neutral $B_{s}$ system will be discussed, while the second section focuses on $B_{S} \rightarrow(J / \psi) \phi$ event reconstruction. Lastly, the prospective results for $1.3 \mathrm{fb}^{-1}$ of integrated luminosity with full Monte Carlo simulation will be shown.

\section{MIXING IN THE NEUTRAL $B_{s}$ SYSTEM}

The $B_{s}$ meson (containing a $\bar{b}$ and an $s$ quark) (Fig.1) oscillates into its anti particle $\bar{B}_{s}$ through weak interaction. By studying $B_{s}-\bar{B}_{s}$ mixing, it is possible to measure parameters, in particular $\Delta m_{s}, \Delta \Gamma_{s}$ and the CP violating phase $\phi_{s}$ [2]. CP violation in the $B_{s}-\bar{B}_{s}$ system is due to interference between decay and mixing.

CP1265, VIII Latin American Symposium on Nuclear Physics and Applications

edited by R. Alcaron, H. F. Arellano, P. L. Cole, and A. J. Kreiner

(C) 2010 American Institute of Physics 978-0-7354-0814-2/10/\$30.00 

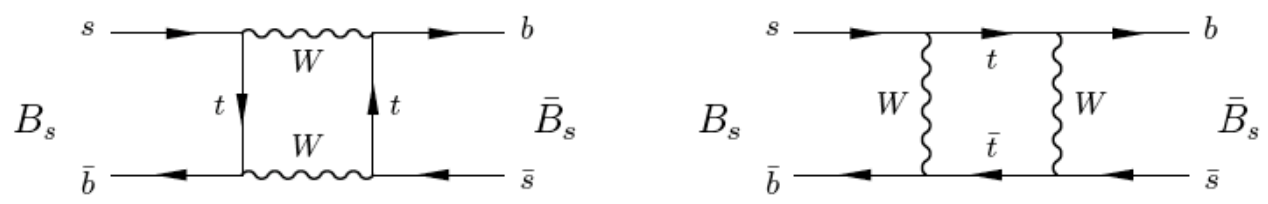

FIGURE 1. The Standard Model permits $B_{s}$ mesons to change into $\bar{B}_{s}$ mesons and vice versa. These transitions lead to particle mixing

The time evolution of the $B_{S}$ and $\bar{B}_{S}$ states can be described by the non-Hermitian Hamiltonian $\mathscr{H}$ which can be represented with $2 \times 2$ matrices

$$
\mathscr{H}=M-\frac{i}{2} \Gamma
$$

The relation between $M, \Gamma$ and the mixing parameters is $\Delta m_{s}=2\left[M_{12}\right]$ and $\Delta \Gamma_{s}=$ $2\left[\Gamma_{12}\right]$, where $\Delta \Gamma_{s}=\Gamma^{H}-\Gamma^{L}$ and $\Delta m_{s}=m_{H}-m_{L}$ and the two eigenstates with heavier and light mass eigenvalues are $B_{s}^{H}$ and $B_{s}^{L}$, respectively.

According to the Standard Model the value for $\phi_{s}$ is -0.04 [3]. However, there are predictions for non-SM value for $\phi_{s}$ due to new physics beyond the SM. Recent results indicate that the $B_{S}$ mixing amplitude deviates by more than $3 \sigma$ from the Standard Model prediction [4].

$$
\begin{gathered}
B_{s} \rightarrow(J / \psi) \phi \rightarrow \mu^{+} \mu^{-} K^{+} K^{-} \text {DECAY CHANNEL } \\
\text { RECONSTRUCTIONS }
\end{gathered}
$$

For the reconstruction of the decay channel, three dedicated trigger levels have been designed. They attempt a reconstruction of the $B_{S} \rightarrow(J / \psi) \phi$ decay chain [5].

A kinematic vertex fit is applied offline and a time-dependent angular analysis is performed to extract the width difference $\Delta \Gamma_{s}$.

To obtain a clean sample of $B_{s} \rightarrow(J / \psi) \phi \rightarrow \mu^{+} \mu^{-} K^{+} K^{-}$events, the following cuts are applied: $p_{T}(\mu)>3 \mathrm{GeV} / \mathrm{c}, P_{T}(J / \psi)>4 \mathrm{GeV} / \mathrm{c}, \Delta m_{J / \psi}<120 \mathrm{MeV} / c^{2}$, and finally $p_{T}(K)>0.8 \mathrm{GeV} / \mathrm{c}, p_{T}(J / \psi)>1 \mathrm{GeV} / \mathrm{c}, \Delta m_{\psi}<20 \mathrm{MeV} / c^{2}$ and $p_{T}\left(B_{S}\right)>5$ $\mathrm{GeV} / \mathrm{c}$, where $\Delta m_{J / \psi}$ and $\Delta m_{\phi}$ denotes the difference between the invariant mass of the reconstructed $(J / \psi)$ and $\phi$ respectively, from the known $(J / \psi)$ mass and $\phi$ mass, 3097 $\mathrm{MeV} / c^{2}$ and $1019 \mathrm{MeV} / c^{2}$ each [6] .

The vertex fit is applied to the two muons tracks and the two kaons tracks, thus constraining the $(J / \psi)$ mass at the same time and improving the resolution of the $B_{S}$ invariant mass from $\sigma=34 \mathrm{MeV} / c^{2}$ to $\sigma=14 \mathrm{MeV} / c^{2}$ (Fig. 2). 


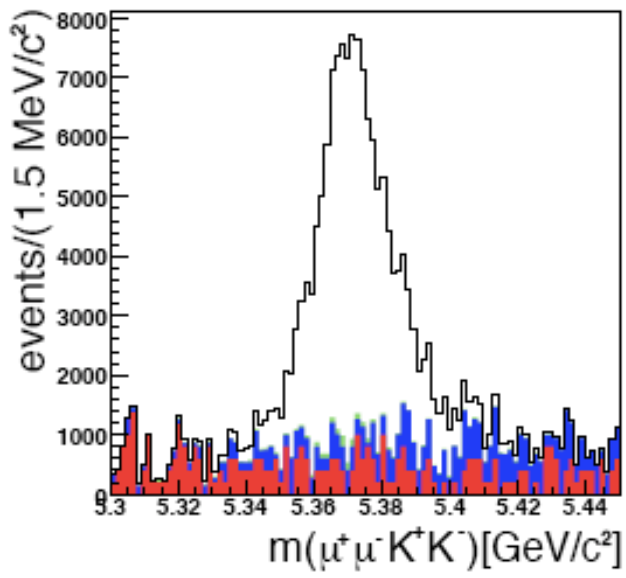

FIGURE 2. Bs invariant mass after cuts: The backgrounds are from inclusive $b \rightarrow(J / \psi) X$ (red), $B_{d} \rightarrow(J / \psi) K^{*}$ (blue) and combinatorial signal events (green).

TABLE 1. Results of the maximum likelihood fit for integrated luminosity of $1.3 \mathrm{fb}^{-1}$ of signal and background at $\sqrt{s}=14 \mathrm{TeV}$

\section{Result Stat. error Total error}

\section{Parameter}

\begin{tabular}{lrrr}
\hline$\left[A_{0}(0)\right]^{2}$ & 0.582 & 0.006 & 0.0163 \\
{$\left[A_{\|}(0)\right]^{2}$} & 0.213 & 0.008 & 0.0099 \\
{$\left[A_{\perp}(0)\right]^{2}$} & 0.205 & 0.007 & 0.0118 \\
$\bar{\Gamma}_{s}$ & $0.7060 \mathrm{ps}^{-1}$ & $0.0080 \mathrm{ps}^{-1}$ & $0.0240 \mathrm{ps}^{-1}$ \\
$\Delta \Gamma_{s}$ & $0.1437 \mathrm{ps}^{-1}$ & $0.026 \mathrm{ps}^{-1}$ & $0.0279 \mathrm{ps}^{-1}$ \\
$\Delta \Gamma_{S} / \bar{\Gamma}_{s}$ & 0.204 & 0.037 & 0.041 \\
\hline
\end{tabular}

\section{Likelihood fit}

The differential decay rate can be expressed as:

$$
\frac{d^{4} \Gamma\left(B_{s}(t)\right)}{d \Theta d t}=f(\Theta, \alpha, t)=\sum_{i=1}^{6} O_{i}(\alpha, t) \cdot g_{i}(\Theta),
$$

indeed $O_{i}$ are kinematics-independent observables and $g_{i}$ the angular distributions. the symbol $\alpha$ represents the set of physical parameters $\left(\Gamma_{L}, \Gamma_{H},\left[A_{0}\right],\left[A_{\|}\right],\left[A_{\perp}\right], \delta_{1}, \delta_{2}, \phi_{S}\right)$, $\Theta$ represents the decay angles which define the kinematics and $t$ is the proper decay time [7].

By applying an unbinned maximum likelihood fit it could be derived the parameters of the $B_{s}$ system shown in Table 1 . 
Due to the tight selection criteria, the background rate is very small. This was verified by considering the following background processes:

1. $B_{d} \rightarrow(J / \psi) K^{*}$ decay,

2. $b \rightarrow(J / \psi) X$ decay,

3. direct $(J / \psi)$ production

\section{CONCLUSIONS}

Prospects for an analysis of $B_{S}$ to $(J / \psi)$ decaying into two muons and $\phi$ decaying into two Kaons events with the CMS detector at LHC have been studied. This decay channel permits the study of different properties of the $B_{s}$ system such as the width difference $\Delta \Gamma_{s}$ between the light and heavy mass eigenstates of the $B_{s}$, and the $\mathrm{CP}$ violating phase $\phi_{s}$. The width difference between the weak eigenstates can be determined with a statistical uncertainty of 0.0011 with $1.3 \mathrm{fb}^{-1}$ of Monte Carlo signal and background of simulated data. Assuming a width difference of $20 \%$, an uncertainty of $4 \%$ is expected for an integrated luminosity of $1.3 \mathrm{fb}^{-1}$.

\section{REFERENCES}

1. C. Amsler et al., "Mechanical Design and Material Budget of the CMS BarrelPixel Detector," doi:10.1088/1748-0221/4/05/P05003, 2009.

2. A. Lenz and U. Nierste, "Theoretical update of Bs-Bs-bar mixing," arXiv:hep-ph/0612167v3, 2007.

3. Alexander Lenz, "Mixing and lifetimes of b-hadrons," doi:10.1063/1.2965074, 2008.

4. The UTfit Collaboration, "First Evidence of New Physics in b-s Transitions," arXiv.org:0803.0659, 2009.

5. V. Ciulli et al., "Study of the decay $B_{s} \rightarrow(J / \psi) \phi \rightarrow \mu^{+} \mu^{-} K^{+} K^{-}$," CMS Note 2006/121, 2006.

6. Particle Data Group Collaboration, C. Amsler et al., Physics Letters B667. doi:10.1016/j.physletb.2008.07.018, 2008.

7. L. Wilke, "Study of the $B_{s}-$ Meson with the First LHC Data," PhD thesis, Zurich University, 2009. 